\title{
Non-cryptographic Primitive for Pseudorandom Permutation
}

\author{
Tetsu Iwata $^{1}$, Tomonobu Yoshino ${ }^{1}$, and Kaoru Kurosawa ${ }^{2}$ \\ 1 Department of Communications and Integrated Systems, \\ Tokyo Institute of Technology \\ 2-12-1 O-okayama, Meguro-ku, Tokyo 152-8552, Japan \\ tez@ss.titech.ac.jp \\ 2 Department of Computer and Information Sciences, \\ Ibaraki University \\ 4-12-1 Nakanarusawa, Hitachi, Ibaraki 316-8511, Japan \\ kurosawa@cis.ibaraki.ac.jp
}

\begin{abstract}
Four round Feistel permutation (like DES) is super-pseudorandom if each round function is random or a secret universal hash function. A similar result is known for five round MISTY type permutation. It seems that each round function must be at least either random or secret in both cases.

In this paper, however, we show that the second round permutation $g$ in five round MISTY type permutation need not be cryptographic at all, i.e., no randomness nor secrecy is required. $g$ has only to satisfy that $g(x) \oplus x \neq g\left(x^{\prime}\right) \oplus x^{\prime}$ for any $x \neq x^{\prime}$. This is the first example such that a non-cryptographic primitive is substituted to construct the minimum round super-pseudorandom permutation. Further we show efficient constructions of super-pseudorandom permutations by using above mentioned $g$.
\end{abstract}

Keywords: Block cipher, pseudorandomness, MISTY type permutation.

\section{Introduction}

\subsection{Super-Pseudorandomness}

A secure block cipher should be indistinguishable from a truly random permutation. Consider an infinitely powerful distinguisher $\mathcal{D}$ which tries to distinguish a block cipher from a truly random permutation. It outputs 0 or 1 after making at most $m$ queries to the given encryption and/or decryption oracles. We say that a distinguisher $\mathcal{D}$ is a pseudorandom distinguisher if it has oracle access to the encryption oracle. We also say that a distinguisher $\mathcal{D}$ is a super-pseudorandom distinguisher if it has oracle access to both the encryption oracle and the decryption oracle. Then a block cipher $E$ is called pseudorandom if any pseudorandom distinguisher $\mathcal{D}$ cannot distinguish $E$ from a truly random permutation. A block cipher $E$ is called super-pseudorandom if any super-pseudorandom distinguisher $\mathcal{D}$ cannot distinguish $E$ from a truly random permutation. 


\subsection{Previous Works}

The super-pseudorandomness of Feistel permutation (like DES) has been studied extensively so far. Let $\phi\left(f_{1}, f_{2}, f_{3}\right)$ denote the three round Feistel permutation such that the $i$-th round function is $f_{i}$. Similarly, let $\phi\left(f_{1}, f_{2}, f_{3}, f_{4}\right)$ denote the four round Feistel permutation.

Suppose that each $f_{i}$ is a random function. Then Luby and Rackoff proved that $\phi\left(f_{1}, f_{2}, f_{3}\right)$ is pseudorandom and $\phi\left(f_{1}, f_{2}, f_{3}, f_{4}\right)$ is super-pseudorandom 4. Lucks showed that the $\phi\left(h_{1}, f_{2}, f_{3}\right)$ is pseudorandom even if $h_{1}$ is an $\epsilon$ XOR universal hash function [5]. Suppose that $h_{1}$ and $h_{4}$ are uniform $\epsilon$-XOR universal hash functions. Then Naor and Reingold proved that $h_{4} \circ \phi\left(f_{2}, f_{3}\right) \circ h_{1}$ is super-pseudorandom [8], and Ramzan and Reyzin showed that $\phi\left(h_{1}, f_{2}, f_{3}, h_{4}\right)$ is super-pseudorandom even if the distinguisher has oracle access to $f_{2}$ and $f_{3}$ [9].

On the other hand, let $\psi\left(p_{1}, p_{2}, p_{3}, p_{4}, p_{5}\right)$ denote the five round MISTY type permutation such that the $i$-th round permutation is $p_{i}$. Suppose that each $p_{i}$ is a random permutation. Then Iwata et al. 3] and Gilbert and Minier 2] independently showed that $\psi\left(p_{1}, p_{2}, p_{3}, p_{4}, p_{5}\right)$ is super-pseudorandom. More than that, let $h_{i}$ be a uniform $\epsilon$-XOR universal permutation. Iwata et al. proved that

1. $\psi\left(h_{1}, h_{2}, p_{3}, p_{4}, h_{5}^{-1}\right)$ is super-pseudorandom even if the distinguisher has oracle access to $p_{3}, p_{3}^{-1}, p_{4}$ and $p_{4}^{-1}$.

2. $\psi\left(h_{1}, p_{2}, p_{3}, p_{4}, h_{5}^{-1}\right)$ is super-pseudorandom even if the distinguisher has oracle access to $p_{2}, p_{2}^{-1}, p_{3}, p_{3}^{-1}, p_{4}$ and $p_{4}^{-1}$.

\subsection{Our Contribution}

Four round Feistel permutation (like DES) is super-pseudorandom if each round function is random or a secret universal hash function. A similar result is known for five round MISTY type permutation. It seems that each round function must be at least either random or secret in both cases.

In this paper, however, we show that the second round permutation $g$ in five round MISTY type permutation need not be cryptographic at all, i.e., no randomness nor secrecy is required. $g$ has only to satisfy that $g(x) \oplus x \neq g\left(x^{\prime}\right) \oplus$ $x^{\prime}$ for any $x \neq x^{\prime}$. This is the first example such that a non-cryptographic primitive is substituted to construct the minimum round super-pseudorandom permutation. Further we show efficient constructions of super-pseudorandom permutations by using above mentioned $g$.

One might wonder if five rounds can be reduced to four rounds to obtain super-pseudorandomness of MISTY. However, it is not true because Sakurai and Zheng showed that the four round MISTY type permutation $\psi\left(p_{1}, p_{2}, p_{3}, p_{4}\right)$ is not super-pseudorandom [10].

More precisely, we prove that five round MISTY is super-pseudorandom if it is $\psi\left(h_{1}, g, p, p^{-1}, h_{5}^{-1}\right)$, where $g$ is the above mentioned permutation, $h_{1}$ is an $\epsilon$ XOR universal permutation, $h_{5}$ is a uniform $\epsilon$-XOR universal permutation, and $p$ is a random permutation. Further, suppose that both $h_{1}$ and $h_{5}$ are uniform 
$\epsilon$-XOR universal permutations. Then we prove that it is super-pseudorandom even if the distinguisher has oracle access to $p$ and $p^{-1}$.

More than that, we study the case such that the third and the fourth round permutations are both $p$. In this case, we show that it is not super-pseudorandom nor pseudorandom if a distinguisher has oracle access to $p$. More formally, we show that for any fixed and public $g, \psi\left(p_{1}, g, p, p, p_{5}\right)$ is not pseudorandom if a distinguisher has oracle access to $p$.

\section{Preliminaries}

\section{$2.1 \quad$ Notation}

For a bit string $x \in\{0,1\}^{2 n}$, we denote the first (left) $n$ bits of $x$ by $x_{L}$ and the last (right) $n$ bits of $x$ by $x_{R}$. If $S$ is a probability space, then $s \stackrel{R}{\leftarrow} S$ denotes the process of picking an element from $S$ according to the underlying probability distribution. The underlying distribution is assumed to be uniform (unless otherwise specified).

Denote by $F_{n}$ the set of all functions from $\{0,1\}^{n}$ to $\{0,1\}^{n}$, which consists of $2^{n \cdot 2^{n}}$ functions in total. Similarly, denote by $P_{n}$ the set of all permutations from $\{0,1\}^{n}$ to $\{0,1\}^{n}$, which consists of $\left(2^{n}\right)$ ! permutations in total.

\subsection{MISTY Type Permutation 67}

Definition 2.1 (The basic MISTY type permutation). Let $x \in\{0,1\}^{2 n}$. For any permutation $p \in P_{n}$, define the basic MISTY type permutation $\psi_{p} \in P_{2 n}$ as $\psi_{p}(x) \stackrel{\text { def }}{=}\left(x_{R}, p\left(x_{L}\right) \oplus x_{R}\right)$. Note that it is a permutation since $\psi_{p}^{-1}(x)=$ $\left(p^{-1}\left(x_{L} \oplus x_{R}\right), x_{L}\right)$.

Definition 2.2 (The $r$ round MISTY type permutation, $\psi$ ). Let $r \geq 1$ be an integer, $p_{1}, \ldots, p_{r} \in P_{n}$ be permutations. Define the $r$ round MISTY type permutation $\psi\left(p_{1}, \ldots, p_{r}\right) \in P_{2 n}$ as $\psi\left(p_{1}, \ldots, p_{r}\right) \stackrel{\text { def }}{=} \rho \circ \psi_{p_{r}} \circ \cdots \circ \psi_{p_{1}}$, where $\rho\left(x_{L}, x_{R}\right)=\left(x_{R}, x_{L}\right)$ for $x \in\{0,1\}^{2 n}$.

See Fig. 1 (the five round MISTY type permutation) for an illustration. Note that $p_{i}$ in Fig. 1 is a permutation. For simplicity, the left and right swaps are omitted.

\subsection{Uniform $\epsilon$-XOR Universal Permutation}

Our definitions follow from those given in 13911 .

Definition 2.3. Let $H_{n}$ be a permutation family over $\{0,1\}^{n}$. Denote by $\# H_{n}$ the size of $H_{n}$. 


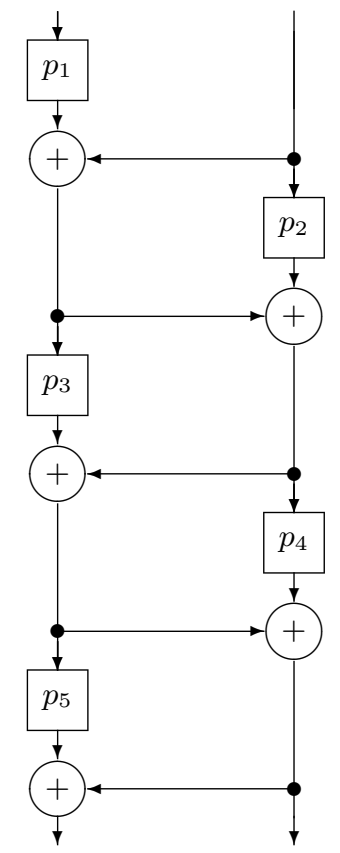

Fig. 1. MISTY type permutation

1. $H_{n}$ is a uniform permutation family if for any element $x \in\{0,1\}^{n}$ and any element $y \in\{0,1\}^{n}$, there exist exactly $\frac{\# H_{n}}{2^{n}}$ permutations $h \in H_{n}$ such that $h(x)=y$.

2. $H_{n}$ is an $\epsilon$-XOR universal permutation family if for any two distinct elements $x, x^{\prime} \in\{0,1\}^{n}$ and any element $y \in\{0,1\}^{n}$, there exist at most $\epsilon \# H_{n}$ permutations $h \in H_{n}$ such that $h(x) \oplus h\left(x^{\prime}\right)=y$.

Let $f_{a}(x) \stackrel{\text { def }}{=} a \cdot x$ over $\mathrm{GF}\left(2^{n}\right)$, where $a \neq 0$. Then $\left\{f_{a}(x)\right\}$ is a $\frac{1}{2^{n}-1}$-XOR universal permutation family.

Let $f_{a, b}(x) \stackrel{\text { def }}{=} a \cdot x+b$ over $\operatorname{GF}\left(2^{n}\right)$, where $a \neq 0$. Then $\left\{f_{a, b}(x)\right\}$ is a uniform $\frac{1}{2^{n}-1}$ XOR universal permutation family.

We will use the phrase " $h$ is an $\epsilon$-XOR universal permutation" to mean that " $h$ is drawn uniformly from an $\epsilon$-XOR universal permutation family". Similarly, we will use the phrase " $h$ is a uniform $\epsilon$-XOR universal permutation".

\section{Improved Super-Pseudorandomness of MISTY Type Permutation}

We say that a permutation $g$ over $\{0,1\}^{n}$ is $X O R$-distinct if

$$
g(x) \oplus x \neq g\left(x^{\prime}\right) \oplus x^{\prime}
$$


for any $x \neq x^{\prime}$. Let $g(x)=a \cdot x$ over $\operatorname{GF}\left(2^{n}\right)$, where $a \neq 0,1$. Then this $g$ is clearly XOR-distinct.

In this section, we prove that $\psi\left(h_{1}, g, p, p^{-1}, h_{5}^{-1}\right)$ is super-pseudorandom even if the second round permutation $g$ is fixed and publicly known. $g$ has only to be XOR-distinct. This means that the five round MISTY type permutation is super-pseudorandom even if the second round permutation has no randomness nor secrecy.

Let $H_{n}^{0}$ be an $\epsilon$-XOR universal permutation family over $\{0,1\}^{n}$, and $H_{n}^{1}$ be a uniform $\epsilon$-XOR universal permutation family over $\{0,1\}^{n}$. Define

$$
\left\{\begin{array}{l}
\operatorname{MISTY}_{2 n}^{01} \stackrel{\text { def }}{=}\left\{\psi\left(h_{1}, g, p, p^{-1}, h_{5}^{-1}\right) \mid p \in P_{n}, h_{1} \in H_{n}^{0}, h_{5} \in H_{n}^{1}\right\} \\
\operatorname{MISTY}_{2 n}^{11} \stackrel{\text { def }}{=}\left\{\psi\left(h_{1}, g, p, p^{-1}, h_{5}^{-1}\right) \mid p \in P_{n}, h_{1}, h_{5} \in H_{n}^{1}\right\}
\end{array}\right.
$$

\subsection{Super-Pseudorandomness of $\operatorname{MISTY}_{2 n}^{01}$}

Let $\mathcal{D}$ be a super-pseudorandom distinguisher for $\operatorname{MISTY}_{2 n}^{01}$ which makes at most $m$ queries in total. We consider two experiments, experiment 0 and experiment 1. In experiment $0, \mathcal{D}$ has oracle access to $\psi$ and $\psi^{-1}$, where $\psi$ is randomly chosen from MISTY M $_{2 n}$. In experiment $1, \mathcal{D}$ has oracle access to $R$ and $R^{-1}$, where $R$ is randomly chosen from $P_{2 n}$.

Define the advantage of $\mathcal{D}$ as follows.

$$
\operatorname{Adv}(\mathcal{D}) \stackrel{\text { def }}{=}\left|p_{\psi}-p_{R}\right|
$$

where

$$
\left\{\begin{array}{l}
p_{\psi} \stackrel{\text { def }}{=} \operatorname{Pr}\left(\mathcal{D}^{\psi, \psi^{-1}}\left(1^{2 n}\right)=1 \mid \psi \stackrel{R}{\leftarrow} \operatorname{MISTY}_{2 n}^{01}\right) \\
p_{R} \stackrel{\text { def }}{=} \operatorname{Pr}\left(\mathcal{D}^{R, R^{-1}}\left(1^{2 n}\right)=1 \mid R \stackrel{R}{\leftarrow} P_{2 n}\right)
\end{array}\right.
$$

Lemma 3.1. Fix $x^{(i)} \in\{0,1\}^{2 n}$ and $y^{(i)} \in\{0,1\}^{2 n}$ for $1 \leq i \leq m$ arbitrarily in such a way that $\left\{x^{(i)}\right\}_{1 \leq i \leq m}$ are all distinct and $\left\{y^{(i)}\right\}_{1 \leq i \leq m}$ are all distinct.

Then the number of $\psi \in \mathrm{MISTY}_{2 n}^{01}$ such that

$$
\psi\left(x^{(i)}\right)=y^{(i)} \text { for } 1 \leq \forall i \leq m
$$

is at least

$$
\left(\# H_{n}^{0}\right)\left(\# H_{n}^{1}\right)\left(2^{n}-2 m\right) !\left(1-2 \epsilon \cdot m(m-1)-\frac{2 m^{2}}{2^{n}}\right)
$$

A proof is given in Appendix A.

Theorem 3.1. For any super-pseudorandom distinguisher $\mathcal{D}$ that makes at most $m$ queries in total,

$$
\operatorname{Adv}(\mathcal{D}) \leq 2 \epsilon \cdot m(m-1)+\frac{2 m^{2}}{2^{n}}
$$


Proof. Let $\mathcal{O}=R$ or $\psi$. The super-pseudorandom distinguisher $\mathcal{D}$ has oracle access to $\mathcal{O}$ and $\mathcal{O}^{-1}$.

There are two types of queries $\mathcal{D}$ can make: either $(+, x)$ which denotes the query "what is $\mathcal{O}(x)$ ?", or $(-, y)$ which denotes the query "what is $\mathcal{O}^{-1}(y)$ ?" For the $i$-th query $\mathcal{D}$ makes to $\mathcal{O}$ or $\mathcal{O}^{-1}$, define the query-answer pair $\left(x^{(i)}, y^{(i)}\right) \in$ $\{0,1\}^{2 n} \times\{0,1\}^{2 n}$, where either $\mathcal{D}$ 's query was $\left(+, x^{(i)}\right)$ and the answer it got was $y^{(i)}$ or $\mathcal{D}$ 's query was $\left(-, y^{(i)}\right)$ and the answer it got was $x^{(i)}$. Define view $v$ of $\mathcal{D}$ as $v=\left(\left(x^{(1)}, y^{(1)}\right), \ldots,\left(x^{(m)}, y^{(m)}\right)\right)$.

Without loss of generality, we assume that $\left\{x^{(i)}\right\}_{1 \leq i \leq m}$ are all distinct, and $\left\{y^{(i)}\right\}_{1 \leq i \leq m}$ are all distinct.

Since $\mathcal{D}$ has unbounded computational power, $\mathcal{D}$ can be assumed to be deterministic. Therefore, the final output of $\mathcal{D}(0$ or 1$)$ depends only on $v$. Hence denote by $\mathcal{C}_{\mathcal{D}}(v)$ the final output of $\mathcal{D}$.

Let $\boldsymbol{v}_{\text {one }} \stackrel{\text { def }}{=}\left\{v \mid \mathcal{C}_{\mathcal{D}}(v)=1\right\}$ and $N_{\text {one }} \stackrel{\text { def }}{=} \# \boldsymbol{v}_{\text {one }}$.

Evaluation of $p_{R}$. From the definition of $p_{R}$, we have

$$
\begin{aligned}
p_{R} & =\operatorname{Pr}\left(\mathcal{D}^{R, R^{-1}}\left(1^{2 n}\right)=1\right) \\
& =\frac{\#\left\{R \mid \mathcal{D}^{R, R^{-1}}\left(1^{2 n}\right)=1\right\}}{\left(2^{2 n}\right) !} .
\end{aligned}
$$

For each $v \in \boldsymbol{v}_{\text {one }}$, the number of $R$ such that

$$
R\left(x^{(i)}\right)=y^{(i)} \text { for } 1 \leq \forall i \leq m
$$

is exactly $\left(2^{2 n}-m\right)$ !. Therefore, we have

$$
\begin{aligned}
p_{R} & =\sum_{v \in \boldsymbol{v}_{\text {one }}} \frac{\#\{R \mid R \text { satisfying (2) }\}}{\left(2^{2 n}\right) !} \\
& =N_{\text {one }} \cdot \frac{\left(2^{2 n}-m\right) !}{\left(2^{2 n}\right) !} .
\end{aligned}
$$

Evaluation of $p_{\psi}$. From the definition of $p_{\psi}$, we have

$$
\begin{aligned}
p_{\psi} & =\operatorname{Pr}_{h_{1}, p, h_{5}}\left(\mathcal{D}^{\psi, \psi^{-1}}\left(1^{2 n}\right)=1\right) \\
& =\frac{\#\left\{\left(h_{1}, p, h_{5}\right) \mid \mathcal{D}^{\psi, \psi^{-1}}\left(1^{2 n}\right)=1\right\}}{\left(\# H_{n}^{0}\right)\left(2^{n}\right) !\left(\# H_{n}^{1}\right)} .
\end{aligned}
$$

Similarly to $p_{R}$, we have

$$
p_{\psi}=\sum_{v \in \boldsymbol{v}_{\text {one }}} \frac{\#\left\{\left(h_{1}, p, h_{5}\right) \mid\left(h_{1}, p, h_{5}\right) \text { satisfying (1) }\right\}}{\left(\# H_{n}^{0}\right)\left(2^{n}\right) !\left(\# H_{n}^{1}\right)} .
$$

Then from Lemma 3.1 we obtain that

$$
p_{\psi} \geq \sum_{v \in \boldsymbol{v}_{\text {one }}} \frac{\left(2^{n}-2 m\right) !\left(1-2 \epsilon \cdot m(m-1)-\frac{2 m^{2}}{2^{n}}\right)}{\left(2^{n}\right) !}
$$




$$
\begin{aligned}
& =N_{\text {one }} \frac{\left(2^{n}-2 m\right) !}{\left(2^{n}\right) !}\left(1-2 \epsilon \cdot m(m-1)-\frac{2 m^{2}}{2^{n}}\right) \\
& =p_{R} \frac{\left(2^{2 n}\right) !\left(2^{n}-2 m\right) !}{\left(2^{2 n}-m\right) !\left(2^{n}\right) !}\left(1-2 \epsilon \cdot m(m-1)-\frac{2 m^{2}}{2^{n}}\right) .
\end{aligned}
$$

Since $\frac{\left(2^{2 n}\right) !\left(2^{n}-2 m\right) !}{\left(2^{2 n}-m\right) !\left(2^{n}\right) !} \geq 1$ (This can be shown easily by an induction on $m$ ), we have

$$
\begin{aligned}
p_{\psi} & \geq p_{R}\left(1-2 \epsilon \cdot m(m-1)-\frac{2 m^{2}}{2^{n}}\right) \\
& \geq p_{R}-2 \epsilon \cdot m(m-1)-\frac{2 m^{2}}{2^{n}} .
\end{aligned}
$$

Applying the same argument to $1-p_{\psi}$ and $1-p_{R}$ yields that

$$
1-p_{\psi} \geq 1-p_{R}-2 \epsilon \cdot m(m-1)-\frac{2 m^{2}}{2^{n}}
$$

Finally, (3) and (44) give $\left|p_{\psi}-p_{R}\right| \leq 2 \epsilon \cdot m(m-1)+\frac{2 m^{2}}{2^{n}}$.

\subsection{Super-Pseudorandomness of MISTY ${ }_{2 n}^{11}$}

Let $\mathcal{D}$ be a super-pseudorandom distinguisher for $\operatorname{MISTY}_{2 n}^{11}$. $\mathcal{D}$ also has oracle access to $p$ and $p^{-1}$, where $p$ and $p^{-1}$ are the third and fourth round permutations of MISTY $_{2 n}^{11}$ respectively. $\mathcal{D}$ makes at most $m$ queries in total. We consider two experiments, experiment 0 and experiment 1 . In experiment $0, \mathcal{D}$ has oracle access to not only $\psi$ and $\psi^{-1}$, but also $p$ and $p^{-1}$, where $\psi$ is randomly chosen from MISTY ${ }_{2 n}^{11}$. In experiment $1, \mathcal{D}$ has oracle access to $R, R^{-1}, p$ and $p^{-1}$, where $R$ is randomly chosen from $P_{2 n}$ and $p$ is randomly chosen from $P_{n}$.

Define the advantage of $\mathcal{D}$ as follows.

$$
\operatorname{Adv}(\mathcal{D}) \stackrel{\text { def }}{=}\left|p_{\psi}-p_{R}\right|
$$

where

$$
\left\{\begin{array}{l}
p_{\psi} \stackrel{\text { def }}{=} \operatorname{Pr}\left(\mathcal{D}^{\psi, \psi^{-1}, p, p^{-1}}\left(1^{2 n}\right)=1 \mid \psi \stackrel{R}{\leftarrow} \operatorname{MISTY}_{2 n}^{11}\right) \\
p_{R} \stackrel{\text { def }}{=} \operatorname{Pr}\left(\mathcal{D}^{R, R^{-1}, p, p^{-1}}\left(1^{2 n}\right)=1 \mid R \stackrel{R}{\leftarrow} P_{2 n}, p \stackrel{R}{\leftarrow} P_{n}\right)
\end{array}\right.
$$

Lemma 3.2. Let $m_{0}$ and $m_{1}$ be integers. Fix $x^{(i)} \in\{0,1\}^{2 n}$ and $y^{(i)} \in\{0,1\}^{2 n}$ for $1 \leq i \leq m_{0}$ arbitrarily in such a way that $\left\{x^{(i)}\right\}_{1 \leq i \leq m_{0}}$ are all distinct and $\left\{y^{(i)}\right\}_{1 \leq i \leq m_{0}}$ are all distinct. Similarly, fix $X^{(i)} \in\{0,1\}^{n}$ and $Y^{(i)} \in\{0,1\}^{n}$ for $1 \leq i \leq m_{1}$ arbitrarily in such a way that $\left\{X^{(i)}\right\}_{1 \leq i \leq m_{1}}$ are all distinct and $\left\{Y^{(i)}\right\}_{1 \leq i \leq m_{1}}$ are all distinct.

Then the number of $\psi \in \operatorname{MISTY}_{2 n}^{11}$ such that

$$
\psi\left(x^{(i)}\right)=y^{(i)} \text { for } 1 \leq \forall i \leq m_{0} \text { and } p\left(X^{(i)}\right)=Y^{(i)} \text { for } 1 \leq \forall i \leq m_{1}
$$

is at least

$$
\left(\# H_{n}^{1}\right)^{2}\left(2^{n}-2 m_{0}-m_{1}\right) !\left(1-2 \epsilon \cdot m_{0}\left(m_{0}-1\right)-\frac{4 m_{0} m_{1}}{2^{n}}-\frac{2 m_{0}^{2}}{2^{n}}\right) .
$$

A proof is given in Appendix B. 
Theorem 3.2. For any super-pseudorandom distinguisher $\mathcal{D}$ that also has oracle access to $p$ and $p^{-1}$ and makes at most $m$ queries in total,

$$
\operatorname{Adv}(\mathcal{D}) \leq 2 \epsilon \cdot m(m-1)+\frac{6 m^{2}}{2^{n}}
$$

Proof. Let $\mathcal{O}=R$ or $\psi$. The super-pseudorandom distinguisher $\mathcal{D}$ has oracle access to $\mathcal{O}, \mathcal{O}^{-1}, p$ and $p^{-1}$. Assume that $\mathcal{D}$ makes $m_{0}$ queries to $\mathcal{O}$ or $\mathcal{O}^{-1}$, and $m_{1}$ queries to $p$ or $p^{-1}$, where $m=m_{0}+m_{1}$.

There are four types of queries $\mathcal{D}$ can make: either $(+, x)$ which denotes the query "what is $\mathcal{O}(x)$ ?", $(-, y)$ which denotes the query "what is $\mathcal{O}^{-1}(y)$ ?", $(+, X)$ which denotes the query "what is $p(X)$ ?", or $(-, Y)$ which denotes the query "what is $p^{-1}(Y)$ ?" For the $i$-th query $\mathcal{D}$ makes to $\mathcal{O}$ or $\mathcal{O}^{-1}$, define the query-answer pair $\left(x^{(i)}, y^{(i)}\right) \in\{0,1\}^{2 n} \times\{0,1\}^{2 n}$, where either $\mathcal{D}$ 's query was $\left(+, x^{(i)}\right)$ and the answer it got was $y^{(i)}$ or $\mathcal{D}$ 's query was $\left(-, y^{(i)}\right)$ and the answer it got was $x^{(i)}$. Similarly for the $i$-th query $\mathcal{D}$ makes to $p$ or $p^{-1}$, define the queryanswer pair $\left(X^{(i)}, Y^{(i)}\right) \in\{0,1\}^{n} \times\{0,1\}^{n}$, where either $\mathcal{D}$ 's query was $\left(+, X^{(i)}\right)$ and the answer it got was $Y^{(i)}$ or $\mathcal{D}$ 's query was $\left(-, Y^{(i)}\right)$ and the answer it got was $X^{(i)}$. Define view $v$ and $V$ of $\mathcal{D}$ as $v=\left(\left(x^{(1)}, y^{(1)}\right), \ldots,\left(x^{\left(m_{0}\right)}, y^{\left(m_{0}\right)}\right)\right)$ and $V=\left(\left(X^{(1)}, Y^{(1)}\right), \ldots,\left(X^{\left(m_{1}\right)}, Y^{\left(m_{1}\right)}\right)\right)$. Without loss of generality, we assume that $\left\{x^{(i)}\right\}_{1 \leq i \leq m_{0}}$ are all distinct, $\left\{y^{(i)}\right\}_{1 \leq i \leq m_{0}}$ are all distinct, $\left\{X^{(i)}\right\}_{1 \leq i \leq m_{1}}$ are all distinct and $\left\{Y^{(i)}\right\}_{1 \leq i \leq m_{1}}$ are all distinct.

Then similarly to the proof of Theorem 3.1, denote by $\mathcal{C}_{\mathcal{D}}(v, V)$ the final output of $\mathcal{D}$.

$$
\text { Let }(\boldsymbol{v}, \boldsymbol{V})_{\text {one }} \stackrel{\text { def }}{=}\left\{(v, V) \mid \mathcal{C}_{\mathcal{D}}(v, V)=1\right\} \text { and } N_{\text {one }} \stackrel{\text { def }}{=} \#(\boldsymbol{v}, \boldsymbol{V})_{\text {one }} \text {. }
$$

Evaluation of $p_{R}$. From the definition of $p_{R}$, we have

$$
\begin{aligned}
p_{R} & =\underset{R, p}{\operatorname{Pr}}\left(\mathcal{D}^{R, R^{-1}, p, p^{-1}}\left(1^{2 n}\right)=1\right) \\
& =\frac{\#\left\{(R, p) \mid \mathcal{D}^{R, R^{-1}, p, p^{-1}}\left(1^{2 n}\right)=1\right\}}{\left(2^{2 n}\right) !\left(2^{n}\right) !} .
\end{aligned}
$$

For each $(v, V) \in(\boldsymbol{v}, \boldsymbol{V})_{\text {one }}$, the number of $(R, p)$ such that

$$
R\left(x^{(i)}\right)=y^{(i)} \text { for } 1 \leq \forall i \leq m_{0} \text { and } p\left(X^{(i)}\right)=Y^{(i)} \text { for } 1 \leq \forall i \leq m_{1}
$$

is exactly $\left(2^{2 n}-m_{0}\right) !\left(2^{n}-m_{1}\right)$ !. Therefore, we have

$$
\begin{aligned}
p_{R} & =\sum_{(v, V) \in(\boldsymbol{v}, \boldsymbol{V})_{\text {one }}} \frac{\#\{(R, p) \mid(R, p) \text { satisfying }(\underline{6})\}}{\left(2^{2 n}\right) !\left(2^{n}\right) !} \\
& =N_{\text {one }} \cdot \frac{\left(2^{2 n}-m_{0}\right) !}{\left(2^{2 n}\right) !} \cdot \frac{\left(2^{n}-m_{1}\right) !}{\left(2^{2 n}\right) !} .
\end{aligned}
$$

Evaluation of $p_{\psi}$. From the definition of $p_{\psi}$, we have

$$
\begin{aligned}
p_{\psi} & =\operatorname{Pr}_{h_{1}, p, h_{5}}\left(\mathcal{D}^{\psi, \psi^{-1}, p, p^{-1}}\left(1^{2 n}\right)=1\right) \\
& =\frac{\#\left\{\left(h_{1}, p, h_{5}\right) \mid \mathcal{D}^{\psi, \psi^{-1}, p, p^{-1}}\left(1^{2 n}\right)=1\right\}}{\left(\# H_{n}^{1}\right)^{2}\left(2^{n}\right) !} .
\end{aligned}
$$

Similarly to $p_{R}$, we have 


$$
p_{\psi}=\sum_{(v, V) \in(\boldsymbol{v}, \boldsymbol{V})_{\text {one }}} \frac{\#\left\{\left(h_{1}, p, h_{5}\right) \mid\left(h_{1}, p, h_{5}\right) \text { satisfying (15) }\right\}}{\left(\# H_{n}^{1}\right)^{2}\left(2^{n}\right) !} .
$$

Then from Lemma 3.2 we obtain that

$$
\begin{aligned}
p_{\psi} & \geq \sum_{(v, V) \in(\boldsymbol{v}, \boldsymbol{V})_{\text {one }}} \frac{\left(2^{n}-2 m_{0}-m_{1}\right) !\left(1-2 \epsilon \cdot m_{0}\left(m_{0}-1\right)-\frac{4 m_{0} m_{1}}{2^{n}}-\frac{2 m_{0}^{2}}{2^{n}}\right)}{\left(2^{n}\right) !} \\
& =N_{\text {one }} \frac{\left(2^{n}-2 m_{0}-m_{1}\right) !}{\left(2^{n}\right) !}\left(1-2 \epsilon \cdot m_{0}\left(m_{0}-1\right)-\frac{4 m_{0} m_{1}}{2^{n}}-\frac{2 m_{0}^{2}}{2^{n}}\right) \\
& =p_{R} \frac{\left(2^{2 n}\right) !\left(2^{n}-2 m_{0}-m_{1}\right) !}{\left(2^{2 n}-m_{0}\right) !\left(2^{n}-m_{1}\right) !}\left(1-2 \epsilon \cdot m_{0}\left(m_{0}-1\right)-\frac{4 m_{0} m_{1}}{2^{n}}-\frac{2 m_{0}^{2}}{2^{n}}\right) .
\end{aligned}
$$

Since $\frac{\left(2^{2 n}\right) !\left(2^{n}-2 m_{0}-m_{1}\right) !}{\left(2^{2 n}-m_{0}\right) !\left(2^{n}-m_{1}\right) !} \geq 1$ (This can be shown easily by an induction on $\left.m_{0}\right)$, we have

$$
\begin{aligned}
p_{\psi} & \geq p_{R}\left(1-2 \epsilon \cdot m_{0}\left(m_{0}-1\right)-\frac{4 m_{0} m_{1}}{2^{n}}-\frac{2 m_{0}^{2}}{2^{n}}\right) \\
& \geq p_{R}-2 \epsilon \cdot m_{0}\left(m_{0}-1\right)-\frac{4 m_{0} m_{1}}{2^{n}}-\frac{2 m_{0}^{2}}{2^{n}} \\
& \geq p_{R}-2 \epsilon \cdot m(m-1)-\frac{6 m^{2}}{2^{n}} .
\end{aligned}
$$

Applying the same argument to $1-p_{\psi}$ and $1-p_{R}$ yields that

$$
1-p_{\psi} \geq 1-p_{R}-2 \epsilon \cdot m(m-1)-\frac{6 m^{2}}{2^{n}}
$$

Finally, (7) and (8) give $\left|p_{\psi}-p_{R}\right| \leq 2 \epsilon \cdot m(m-1)+\frac{6 m^{2}}{2^{n}}$.

\section{Negative Result}

Let $g$ be a fixed and publicly known XOR-distinct permutation. In Theorem 3.2, we showed that $\psi\left(h_{1}, g, p, p^{-1}, h_{5}^{-1}\right)$ is super-pseudorandom even if the distinguisher has oracle access to $p$ and $p^{-1}$, where $h_{1}$ and $h_{5}$ are uniform $\epsilon$-XOR universal permutations, and $p$ is a random permutation.

One might think that $\psi\left(h_{1}, g, p, p, h_{5}^{-1}\right)$ is super-pseudorandom even if the distinguisher has oracle access to $p$ and $p^{-1}$. In this section, however, we show that this is not true. We can distinguish $\psi\left(h_{1}, g, p, p, h_{5}^{-1}\right)$ from a random permutation with advantage very close to 1 .

More generally, let $p_{1}, p_{2}, p, p_{5} \in P_{n}$ be random permutations and $\psi=$ $\psi\left(p_{1}, p_{2}, p, p, p_{5}\right)$. We prove that $\psi$ is not pseudorandom if the distinguisher has oracle access to $p_{2}, p_{2}^{-1}$ and $p$. This proof implies that for any fixed and public $g, \psi\left(p_{1}, g, p, p, p_{5}\right)$ is not super-pseudorandom nor pseudorandom if the distinguisher has oracle access to $p$. 
Define the advantage of $\mathcal{D}$ as follows.

$$
\operatorname{Adv}(\mathcal{D}) \stackrel{\text { def }}{=}\left|p_{\psi}-p_{R}\right|
$$

where

$$
\left\{\begin{array}{l}
p_{\psi} \stackrel{\text { def }}{=} \operatorname{Pr}\left(\mathcal{D}^{\psi, p_{2}, p_{2}^{-1}, p}\left(1^{2 n}\right)=1 \mid p_{1}, p_{2}, p, p_{5} \stackrel{R}{\leftarrow} P_{n}, \psi=\psi\left(p_{1}, p_{2}, p, p, p_{5}\right)\right) \\
p_{R} \stackrel{\text { def }}{=} \operatorname{Pr}\left(\mathcal{D}^{R, p_{2}, p_{2}^{-1}, p}\left(1^{2 n}\right)=1 \mid R \stackrel{R}{\leftarrow} P_{2 n}, p_{2}, p \stackrel{R}{\leftarrow} P_{n}\right)
\end{array}\right.
$$

Theorem 4.1. There exists a pseudorandom distinguisher $\mathcal{D}$ that has oracle access to $p_{2}, p_{2}^{-1}$ and $p$ and makes 6 queries in total,

$$
\operatorname{Adv}(\mathcal{D}) \geq 1-\frac{2}{2^{n}}
$$

Proof. Let $\mathcal{O}=R$ or $\psi$. Our distinguisher $\mathcal{D}$ has oracle access to $\mathcal{O}, p_{2}, p_{2}^{-1}$ and p. Consider the following $\mathcal{D}$ :

1. Ask $(0, \ldots, 0) \in\{0,1\}^{n}$ to $p_{2}^{-1}$ and obtain $A$.

2. Pick $X, A^{\prime} \in\{0,1\}^{n}$ such that $A \neq A^{\prime}$ arbitrarily.

3. Ask $(X, A)$ to $\mathcal{O}$ and obtain $(Y, B)$.

4. Ask $A \oplus A^{\prime}$ to $p_{2}$ and obtain $C$.

5. Ask $A^{\prime} \oplus B$ to $p$ and obtain $D$.

6. Ask $A^{\prime} \oplus B \oplus C$ to $p$ and obtain $E$.

7. Ask $\left(X, A \oplus A^{\prime}\right)$ to $\mathcal{O}$ and obtain $(Z, F)$.

8. Output " 1 " if and only if $F=A^{\prime} \oplus B \oplus C \oplus D \oplus E$.

If $\mathcal{O}=\psi$, then $B$ is the input to $p$ in both third round and fourth round at step 3 since $p_{2}(A)=(0, \ldots, 0)$. Therefore we have $p_{1}(X) \oplus A=B$. Now the input to $p$ in the third round at step 7 is $p_{1}(X) \oplus A \oplus A^{\prime}$ which is equivalent to $A^{\prime} \oplus B$. Next the input to $p$ in the fourth round at step 7 is $A^{\prime} \oplus B \oplus C$ since $p_{2}\left(A \oplus A^{\prime}\right)=C$. Then we always have $F=A^{\prime} \oplus B \oplus C \oplus D \oplus E$ at step 8 . Hence we have $p_{\psi}=1$.

If $\mathcal{O}=R$, we have $p_{R}=\frac{2^{n}}{2^{2 n}-1} \leq \frac{2}{2^{n}}$.

Corollary 4.1. For any fixed and public $g, \psi\left(p_{1}, g, p, p, p_{5}\right)$ is not super-pseudorandom if the distinguisher has oracle access to $p$.

Proof. From the proof of Theorem 4.1

\section{Conclusion}

In this paper, we proposed more efficient constructions of super-pseudorandom permutations based on the five round MISTY type permutation than those given in [3].

In particular, we showed that the second round permutation $g$ need not be cryptographic at all, i.e., no randomness nor secrecy is required.

More precisely, let $p$ and $p_{i}$ be random permutations, then we proved that 
1. $\psi\left(h_{1}, g, p, p^{-1}, h_{5}^{-1}\right)$ is super-pseudorandom, where $h_{1}$ is an $\epsilon$-XOR universal permutation, $g$ is a (publicly known and fixed) XOR-distinct permutation, and $h_{5}$ is a uniform $\epsilon$-XOR universal permutation (Theorem 3.1),

2. $\psi\left(h_{1}, g, p, p^{-1}, h_{5}^{-1}\right)$ is super-pseudorandom, even if the adversary has oracle access to $p$ and $p^{-1}$, where $h_{1}$ and $h_{5}$ are uniform $\epsilon$-XOR universal permutations, and $g$ is a (publicly known and fixed) XOR-distinct permutation (Theorem 3.2),

3. but $\psi\left(p_{1}, p_{2}, p, p, p_{5}\right)$ is not pseudorandom nor super-pseudorandom, if the adversary has oracle access to $p_{2}, p_{2}^{-1}$ and $p$ (Theorem 4.1).

\section{References}

1. J. L. Carter and M. N. Wegman. Universal classes of hash functions. JCSS, vol. 18, no. 2, pp. 143-154, 1979.

2. H. Gilbert and M. Minier. New results on the pseudorandomness of some block cipher constructions. Pre-proceedings of Fast Software Encryption, FSE 2001, pp. 260-277 (to appear in LNCS, Springer-Verlag).

3. T. Iwata, T. Yoshino, T. Yuasa and K. Kurosawa. Round security and superpseudorandomness of MISTY type structure. Pre-proceedings of Fast Software Encryption, FSE 2001, pp. 245-259 (to appear in LNCS, Springer-Verlag).

4. M. Luby and C. Rackoff. How to construct pseudorandom permutations from pseudorandom functions. SIAM J. Comput., vol. 17, no. 2, pp. 373-386, April 1988.

5. S. Lucks. Faster Luby-Rackoff ciphers. Fast Software Encryption, FSE '96, LNCS 1039, pp. 189-203, Springer-Verlag.

6. M. Matsui. New structure of block ciphers with provable security against differential and linear cryptanalysis. Fast Software Encryption, FSE '96, LNCS 1039, pp. 206-218, Springer-Verlag.

7. M. Matsui. New block encryption algorithm MISTY. Fast Software Encryption, FSE '97, LNCS 1267, pp. 54-68, Springer-Verlag.

8. M. Naor and O. Reingold. On the construction of pseudorandom permutations: Luby-Rackoff revised. J. Cryptology, vol. 12, no. 1, pp. 29-66, Springer-Verlag, 1999.

9. Z. Ramzan and L. Reyzin. On the round security of symmetric-key cryptographic primitives. Advances in Cryptology - CRYPTO 2000, LNCS 1880, pp. 376-393, Springer-Verlag, 2000.

10. K. Sakurai and Y. Zheng. On non-pseudorandomness from block ciphers with provable immunity against linear cryptanalysis. IEICE Trans. Fundamentals, vol. E80-A, no. 1, pp. 19-24, April 1997.

11. M. N. Wegman and J. L. Carter. New hash functions and their use in authentication and set equality. JCSS, vol. 22, no. 3, pp. 265-279, 1981.

\section{Appendix A. Proof of Lemma 3.1}

In $\psi$, we denote by $I_{3}^{(i)} \in\{0,1\}^{n}$ the input to $p$ in the third round, and denote by $O_{3}^{(i)} \in\{0,1\}^{n}$ the output of it. Similarly, $I_{4}^{(i)}, O_{4}^{(i)} \in\{0,1\}^{n}$ are the input 
and output of $p$ in the fourth round, respectively. That is, $p\left(I_{3}^{(i)}\right)=O_{3}^{(i)}$ and $p\left(I_{4}^{(i)}\right)=O_{4}^{(i)}$.

Number of $h_{1}$. First, for any fixed $i$ and $j$ such that $1 \leq i<j \leq m$ :

- if $x_{L}^{(i)}=x_{L}^{(j)}$, then there exists no $h_{1}$ such that

$$
h_{1}\left(x_{L}^{(i)}\right) \oplus x_{R}^{(i)}=h_{1}\left(x_{L}^{(j)}\right) \oplus x_{R}^{(j)}
$$

since $x_{L}^{(i)}=x_{L}^{(j)}$ implies $x_{R}^{(i)} \neq x_{R}^{(j)}$;

- if $x_{L}^{(i)} \neq x_{L}^{(j)}$, then the number of $h_{1}$ which satisfies (9) is at most $\epsilon \# H_{n}^{0}$ since $h_{1}$ is an $\epsilon$-XOR universal permutation.

Therefore, the number of $h_{1}$ such that

$$
h_{1}\left(x_{L}^{(i)}\right) \oplus x_{R}^{(i)}=h_{1}\left(x_{L}^{(j)}\right) \oplus x_{R}^{(j)} \text { for } 1 \leq \exists i<\exists j \leq m
$$

is at most $\epsilon\left(\begin{array}{c}m \\ 2\end{array}\right) \# H_{n}^{0}$.

Next, for any fixed $i$ and $j$ such that $1 \leq i<j \leq m$ :

- if $x_{L}^{(i)}=x_{L}^{(j)}$, then there exists no $h_{1}$ such that

$$
h_{1}\left(x_{L}^{(i)}\right) \oplus g\left(x_{R}^{(i)}\right) \oplus x_{R}^{(i)}=h_{1}\left(x_{L}^{(j)}\right) \oplus g\left(x_{R}^{(j)}\right) \oplus x_{R}^{(j)}
$$

since $x_{L}^{(i)}=x_{L}^{(j)}$ implies $x_{R}^{(i)} \neq x_{R}^{(j)}$, and our XOR-distinct $g$ guarantees $g\left(x_{R}^{(i)}\right) \oplus x_{R}^{(i)} \neq g\left(x_{R}^{(j)}\right) \oplus x_{R}^{(j)}$;

- if $x_{L}^{(i)} \neq x_{L}^{(j)}$, then the number of $h_{1}$ which satisfies 11 is at most $\epsilon \# H_{n}^{0}$ since $h_{1}$ is an $\epsilon$-XOR universal permutation.

Therefore, the number of $h_{1}$ such that

$$
h_{1}\left(x_{L}^{(i)}\right) \oplus g\left(x_{R}^{(i)}\right) \oplus x_{R}^{(i)}=h_{1}\left(x_{L}^{(j)}\right) \oplus g\left(x_{R}^{(j)}\right) \oplus x_{R}^{(j)} \text { for } 1 \leq \exists i<\exists j \leq m
$$

is at most $\epsilon\left(\begin{array}{c}m \\ 2\end{array}\right) \# H_{n}^{0}$.

Then, from (10) and (12), the number of $h_{1}$ such that

$$
\left.\begin{array}{l}
h_{1}\left(x_{L}^{(i)}\right) \oplus x_{R}^{(i)} \neq h_{1}\left(x_{L}^{(j)}\right) \oplus x_{R}^{(j)} \text { for } 1 \leq \forall i<\forall j \leq m, \text { and } \\
h_{1}\left(x_{L}^{(i)}\right) \oplus g\left(x_{R}^{(i)}\right) \oplus x_{R}^{(i)} \neq h_{1}\left(x_{L}^{(j)}\right) \oplus g\left(x_{R}^{(j)}\right) \oplus x_{R}^{(j)} \text { for } 1 \leq \forall i<\forall j \leq m
\end{array}\right\}
$$

is at least $\# H_{n}^{0}-2 \epsilon\left(\begin{array}{c}m \\ 2\end{array}\right) \# H_{n}^{0}$. Fix $h_{1}$ which satisfies (13) arbitrarily. This implies that $I_{3}^{(1)}, \ldots, I_{3}^{(m)}$ and $O_{4}^{(1)}, \ldots, O_{4}^{(m)}$ are fixed in such a way that:

$-I_{3}^{(i)} \neq I_{3}^{(j)}$ for $1 \leq \forall i<\forall j \leq m$, and

$-O_{4}^{(i)} \neq O_{4}^{(j)}$ for $1 \leq \forall i<\forall j \leq m$. 
Number of $h_{5}$. Similarly, the number of $h_{5}$ such that

$$
\left.\begin{array}{l}
h_{5}\left(y_{L}^{(i)} \oplus y_{R}^{(i)}\right) \oplus y_{R}^{(i)} \neq h_{5}\left(y_{L}^{(j)} \oplus y_{R}^{(j)}\right) \oplus y_{R}^{(j)} \text { for } 1 \leq \forall i<\forall j \leq m, \\
h_{5}\left(y_{L}^{(i)} \oplus y_{R}^{(i)}\right) \oplus O_{4}^{(i)} \neq h_{5}\left(y_{L}^{(j)} \oplus y_{R}^{(j)}\right) \oplus O_{4}^{(j)} \text { for } 1 \leq \forall i<\forall j \leq m, \\
h_{5}\left(y_{L}^{(i)} \oplus y_{R}^{(i)}\right) \oplus O_{4}^{(i)} \neq O_{4}^{(j)} \text { for } 1 \leq \forall i, \forall j \leq m, \text { and } \\
h_{5}\left(y_{L}^{(i)} \oplus y_{R}^{(i)}\right) \oplus y_{R}^{(i)} \neq I_{3}^{(j)} \text { for } 1 \leq \forall i, \forall j \leq m,
\end{array}\right\}
$$

is at least $\# H_{n}^{1}-2 \epsilon\left(\begin{array}{c}m \\ 2\end{array}\right) \# H_{n}^{1}-\frac{2 m^{2} \# H_{n}^{1}}{2^{n}}$. Fix $h_{5}$ which satisfies (14) arbitrarily. This implies that $O_{3}^{(1)}, \ldots, O_{3}^{(m)}$ and $I_{4}^{(1)}, \ldots, I_{4}^{(m)}$ are fixed in such a way that:

$-I_{4}^{(i)} \neq I_{4}^{(j)}$ for $1 \leq \forall i<\forall j \leq m$,

$-O_{3}^{(i)} \neq O_{3}^{(j)}$ for $1 \leq \forall i<\forall j \leq m$,

$-O_{3}^{(i)} \neq O_{4}^{(j)}$ for $1 \leq \forall i, \forall j \leq m$, and

$-I_{4}^{(i)} \neq I_{3}^{(j)}$ for $1 \leq \forall i, \forall j \leq m$.

Number of $p$. Now $h_{1}$ and $h_{5}$ are fixed in such a way that

$$
I_{3}^{(1)}, \ldots, I_{3}^{(m)}, I_{4}^{(1)}, \ldots, I_{4}^{(m)}
$$

(which are inputs to $p$ ) are all distinct and

$$
O_{3}^{(1)}, \ldots, O_{3}^{(m)}, O_{4}^{(1)}, \ldots, O_{4}^{(m)}
$$

(which are corresponding outputs of $p$ ) are all distinct. In other words, for $p$, the above $2 m$ input-output pairs are determined. The other $2^{n}-2 m$ input-output pairs are undetermined. Therefore we have $\left(2^{n}-2 m\right)$ ! possible choice of $p$ for any such fixed $h_{1}$ and $h_{5}$.

To summarize, we have:

- at least $\# H_{n}^{0}-2 \epsilon\left(\begin{array}{c}m \\ 2\end{array}\right) \# H_{n}^{0}$ choice of $h_{1}$,

- at least \# $H_{n}^{1}-2 \epsilon\left(\begin{array}{c}m \\ 2\end{array}\right) \# H_{n}^{1}-\frac{2 m^{2} \# H_{n}^{1}}{2^{n}}$ choice of $h_{5}$ when $h_{1}$ is fixed, and

- $\left(2^{n}-2 m\right)$ ! choice of $p$ when $h_{1}$ and $h_{5}$ are fixed.

Then the number of $\psi \in \operatorname{MISTY}_{2 n}^{01}$ which satisfy (1) is at least

$$
\begin{gathered}
\left(\# H_{n}^{0}\right)\left(\# H_{n}^{1}\right)\left(2^{n}-2 m\right) !\left(1-2 \epsilon\left(\begin{array}{c}
m \\
2
\end{array}\right)\right)\left(1-2 \epsilon\left(\begin{array}{c}
m \\
2
\end{array}\right)-\frac{2 m^{2}}{2^{n}}\right) \\
\geq\left(\# H_{n}^{0}\right)\left(\# H_{n}^{1}\right)\left(2^{n}-2 m\right) !\left(1-2 \epsilon \cdot m(m-1)-\frac{2 m^{2}}{2^{n}}\right)
\end{gathered}
$$

This concludes the proof of the lemma. 


\section{Appendix B. Proof of Lemma 3.2}

We use the same definition of $I_{3}^{(i)}, O_{3}^{(i)}, I_{4}^{(i)}$ and $O_{4}^{(i)}$ as in the proof of Lemma 3.1 .

Number of $h_{1}$. First, similarly to the proof of Lemma 3.1 the number of $h_{1}$ such that

$$
\begin{aligned}
& h_{1}\left(x_{L}^{(i)}\right) \oplus x_{R}^{(i)} \neq h_{1}\left(x_{L}^{(j)}\right) \oplus x_{R}^{(j)} \text { for } 1 \leq \forall i<\forall j \leq m_{0}, \\
& h_{1}\left(x_{L}^{(i)}\right) \oplus x_{R}^{(i)} \neq X^{(j)} \text { for } 1 \leq \forall i \leq m_{0} \text { and } 1 \leq \forall j \leq m_{1}, \\
& h_{1}\left(x_{L}^{(i)}\right) \oplus g\left(x_{R}^{(i)}\right) \oplus x_{R}^{(i)} \neq h_{1}\left(x_{L}^{(j)}\right) \oplus g\left(x_{R}^{(j)}\right) \oplus x_{R}^{(j)} \text { for } 1 \leq \forall i<\forall j \leq m_{0}, \\
& h_{1}\left(x_{L}^{(i)}\right) \oplus g\left(x_{R}^{(i)}\right) \oplus x_{R}^{(i)} \neq Y^{(j)} \text { for } 1 \leq \forall i \leq m_{0} \text { and } 1 \leq \forall j \leq m_{1}
\end{aligned}
$$

is at least $\# H_{n}^{1}-2 \epsilon\left(\begin{array}{c}m_{0} \\ 2\end{array}\right) \# H_{n}^{1}-\frac{2 m_{0} m_{1} \# H_{n}^{1}}{2^{n}}$. Fix $h_{1}$ which satisfies (15) arbitrarily. This implies that $I_{3}^{(1)}, \ldots, I_{3}^{\left(m_{0}\right)}$ and $O_{4}^{(1)}, \ldots, O_{4}^{\left(m_{0}\right)}$ are fixed in such a way that:

$-I_{3}^{(i)} \neq I_{3}^{(j)}$ for $1 \leq \forall i<\forall j \leq m_{0}$,

$-I_{3}^{(i)} \neq X^{(j)}$ for $1 \leq \forall i \leq m_{0}$ and $1 \leq \forall j \leq m_{1}$,

$-O_{4}^{(i)} \neq O_{4}^{(j)}$ for $1 \leq \forall i<\forall j \leq m_{0}$, and

$-O_{4}^{(i)} \neq Y^{(j)}$ for $1 \leq \forall i \leq m_{0}$ and $1 \leq \forall j \leq m_{1}$.

Number of $h_{5}$. Similarly, the number of $h_{5}$ such that

$$
\left.\begin{array}{l}
h_{5}\left(y_{L}^{(i)} \oplus y_{R}^{(i)}\right) \oplus y_{R}^{(i)} \neq h_{5}\left(y_{L}^{(j)} \oplus y_{R}^{(j)}\right) \oplus y_{R}^{(j)} \text { for } 1 \leq \forall i<\forall j \leq m_{0}, \\
h_{5}\left(y_{L}^{(i)} \oplus y_{R}^{(i)}\right) \oplus y_{R}^{(i)} \neq X^{(j)} \text { for } 1 \leq \forall i \leq m_{0} \text { and } 1 \leq \forall j \leq m_{0}, \\
h_{5}\left(y_{L}^{(i)} \oplus y_{R}^{(i)}\right) \oplus O_{4}^{(i)} \neq h_{5}\left(y_{L}^{(j)} \oplus y_{R}^{(j)}\right) \oplus O_{4}^{(j)} \text { for } 1 \leq \forall i<\forall j \leq m_{0}, \\
h_{5}\left(y_{L}^{(i)} \oplus y_{R}^{(i)}\right) \oplus O_{4}^{(i)} \neq Y^{(j)} \text { for } 1 \leq \forall i \leq m_{0} \text { and } 1 \leq \forall j \leq m_{0}, \\
h_{5}\left(y_{L}^{(i)} \oplus y_{R}^{(i)}\right) \oplus O_{4}^{(i)} \neq O_{4}^{(j)} \text { for } 1 \leq \forall i, \forall j \leq m_{0}, \text { and } \\
h_{5}\left(y_{L}^{(i)} \oplus y_{R}^{(i)}\right) \oplus y_{R}^{(i)} \neq I_{3}^{(j)} \text { for } 1 \leq \forall i, \forall j \leq m_{0},
\end{array}\right\}
$$

is at least $\# H_{n}^{1}-2 \epsilon\left(\begin{array}{c}m_{0} \\ 2\end{array}\right) \# H_{n}^{1}-\frac{2 m_{0} m_{1} \# H_{n}^{1}}{2^{n}}-\frac{2 m_{0}^{2} \# H_{n}^{1}}{2^{n}}$. Fix $h_{5}$ which satisfies (16) arbitrarily. This implies that $O_{3}^{(1)}, \ldots, O_{3}^{\left(m_{0}\right)}$ and $I_{4}^{(1)}, \ldots, I_{4}^{\left(m_{0}\right)}$ are fixed in such a way that:

$-I_{4}^{(i)} \neq I_{4}^{(j)}$ for $1 \leq \forall i<\forall j \leq m_{0}$,

$-I_{4}^{(i)} \neq X^{(j)}$ for $1 \leq \forall i \leq m_{0}$ and $1 \leq \forall j \leq m_{1}$,

$-O_{3}^{(i)} \neq O_{3}^{(j)}$ for $1 \leq \forall i<\forall j \leq m_{0}$,

$-O_{3}^{(i)} \neq Y^{(j)}$ for $1 \leq \forall i \leq m_{0}$ and $1 \leq \forall j \leq m_{1}$,

$-O_{3}^{(i)} \neq O_{4}^{(j)}$ for $1 \leq \forall i, \forall j \leq m_{0}$, and

$-I_{4}^{(i)} \neq I_{3}^{(j)}$ for $1 \leq \forall i, \forall j \leq m_{0}$.

Number of $p$. Now $h_{1}$ and $h_{5}$ are fixed in such a way that

$$
I_{3}^{(1)}, \ldots, I_{3}^{\left(m_{0}\right)}, I_{4}^{(1)}, \ldots, I_{4}^{\left(m_{0}\right)}, X^{(1)}, \ldots, X^{\left(m_{1}\right)}
$$

(which are inputs to $p$ ) are all distinct and 


$$
O_{3}^{(1)}, \ldots, O_{3}^{\left(m_{0}\right)}, O_{4}^{(1)}, \ldots, O_{4}^{\left(m_{0}\right)}, Y^{(1)}, \ldots, Y^{\left(m_{1}\right)}
$$

(which are corresponding outputs of $p$ ) are all distinct. Then we have $\left(2^{n}-\right.$ $\left.2 m_{0}-m_{1}\right)$ ! possible choice of $p$ for any such fixed $h_{1}$ and $h_{5}$.

To summarize, we have:

- at least $\# H_{n}^{1}-2 \epsilon\left(\begin{array}{c}m_{0} \\ 2\end{array}\right) \# H_{n}^{1}-\frac{2 m_{0} m_{1} \# H_{n}^{1}}{2^{n}}$ choice of $h_{1}$,

- at least \# $H_{n}^{1}-2 \epsilon\left(\begin{array}{c}m_{0} \\ 2\end{array}\right) \# H_{n}^{1}-\frac{2 m_{0} m_{1} \# H_{n}^{1}}{2^{n}}-\frac{2 m_{0}^{2} \# H_{n}^{1}}{2^{n}}$ choice of $h_{5}$ when $h_{1}$ is fixed, and

$-\left(2^{n}-2 m_{0}-m_{1}\right)$ ! choice of $p$ when $h_{1}$ and $h_{5}$ are fixed.

Then the number of $\psi \in \operatorname{MISTY}_{2 n}^{11}$ which satisfy (5) is at least

$$
\begin{aligned}
& \left(\# H_{n}^{1}\right)^{2}\left(2^{n}-2 m_{0}-m_{1}\right) ! \\
& \quad \times\left(1-2 \epsilon\left(\begin{array}{c}
m_{0} \\
2
\end{array}\right)-\frac{2 m_{0} m_{1}}{2^{n}}\right)\left(1-2 \epsilon\left(\begin{array}{c}
m_{0} \\
2
\end{array}\right)-\frac{2 m_{0} m_{1}}{2^{n}}-\frac{2 m_{0}^{2}}{2^{n}}\right) \\
& \geq\left(\# H_{n}^{1}\right)^{2}\left(2^{n}-2 m_{0}-m_{1}\right) !\left(1-2 \epsilon \cdot m_{0}\left(m_{0}-1\right)-\frac{4 m_{0} m_{1}}{2^{n}}-\frac{2 m_{0}^{2}}{2^{n}}\right)
\end{aligned}
$$

This concludes the proof of the lemma. 